\title{
RIEMANN SURFACES, OPERATOR FIELDS, STRINGS. ANALOGUES OF THE FOURIER-LAURENT BASES
}

\author{
I.M. KRICHEVER AND S.P. NOVIKOV
}

In the previous works [1-3], started in 1986, the grounds of the operator theory of the interacting closed bosonic strings were constructed. Our theory is the direct development of the classical algebraic operator theory of non-interacting bosonic string of Virasoro. Mandelstam et. al. [4-5]. This theory corresponds to the case of the Riemann surface of the genus $g=0$. The approach which we proposed unifies the ideas of the operator formalism with the ideas of the geometrical approach of Polyakov and others [6-8].

This paper is a brief review and an extension of the works [1-3].

\section{Fourier-Laurent-type bases on the Riemann surfaces. Almost GRADED ANAlOgues OF THE HEIZENBERG AND Virasoro algebras}

The classical definition of the "Heizenberg algebra" (the algebra of the creation and annihilation operators for the non-interacting bosonic string) uses the Fourier expansions of the co-ordinates $X^{\mu}(\sigma)$ and momentums $P^{\mu}(\sigma), 0<\sigma \leqslant 2 \pi, \mu=$ $1, \ldots, \mathcal{D}$. It turns out that this definition can be easily generalized for the case of the interacting string in which the world sheet can be a surface of an arbitrary genus. To obtain this generalization it is sufficient to introduce the special FourierLaurent-type bases, which are defined by the surface itself.

Let's consider a nonsingular Riemann surface $\Gamma$ of a genus $g$ with punctures $P_{i}$ and with fixed real numbers $p_{i}$ such that

$$
\sum_{i=1}^{m} p_{i}=0 \text {. }
$$

The set of data $\left(\Gamma, P_{i}, p_{i}\right)$ will be called the "multistring diagram". For any such diagram there exists a unique differential $d k$ which satisfies the following properties: a) it is holomorphic on $\Gamma$ outside the punctures $P_{i}$; b) at every point $P_{i}$ it has a simple pole with the residue equal to $p_{i}$; c) the periods of $d k$ over an arbitrary closed cycle $\gamma$ on $\Gamma$ are pure imaginary, i.e.

$$
\operatorname{Re} \int_{\gamma} d k=0
$$

From the last condition it follows that the real part of the multivalued function $k(z)$ is single-valued. This function $\tau(z)=\operatorname{Re} k(z)$ is called the "euclidian time". We denote the curves $\tau(z)=$ const $=\tau$ by $C_{\tau}$ and the domains $\tau_{1} \leqslant \tau(z) \leqslant \tau_{2}$ by

Date: April 1989 
$C_{\tau_{1} \tau_{2}}$ (Riemann annulus). The curves $c_{\tau}$ for $x \rightarrow \pm \infty$ tend to small circles around the points $P_{\alpha}$ for which $p_{\alpha}<0$ and $p_{\alpha}>0$, respectively. The transformation $p_{\alpha} \rightarrow$ $a p_{\alpha}$ corresponds to the transformation $\tau \rightarrow a \tau$, which preserves the "chronological ordering" in the case $a>0$. That's why in the simplest case of "one-string" diagram $\left(\Gamma, P_{ \pm}\right)$we can always assume that $p_{+}=1, p_{-}=-1$.

For any integer $\lambda \neq 0,1$ and "one-string" diagram $\left(\Gamma, P_{ \pm}\right)$in the general position, $g \neq 1$, for any integer $n+g / 2$ there exists the unique up to a constant factor tensor $f_{n}^{\lambda}=f_{n}^{\lambda}(z)$ of the weight $\lambda$ with the following analytical properties: a) it is holomorphic on $\Gamma$ outside the points $P_{ \pm}$; b) it has the form

$$
f_{n}^{\lambda}=\varphi_{n, \lambda}^{ \pm} z_{ \pm}^{ \pm n-S}\left(1+O\left(z_{ \pm}\right)\right)\left(d z_{ \pm}\right)^{\lambda}, \quad S=g / 2-\lambda(g-1),
$$

near the points $P_{ \pm}$. Here $z_{ \pm}=z_{ \pm}(Q)$ are local co-ordinates in the neighbourhoods of the points $P_{ \pm}$respectively.

For the exceptional cases ( $g>1, \lambda=0,1 ; g=1, \lambda$-arbitrary integer) the tensor fields $f_{n}^{\lambda}$ are defined by the conditions (1.3) for all except the finite number of $n$ (see $[1,2])$. For other $n \in Z-g / 2$ the definition of $f_{n}^{\lambda}$ is slightly more complicated. We don't present them here because their exact form is not essential for us now.

The bases $f_{n}^{\lambda}$ and $f_{1-\lambda}^{m}$ is dual to each other

$$
\frac{1}{2 \pi i} \cdot \int_{C_{\tau}} f_{n}^{\lambda} \cdot f_{1-\lambda}^{m}=\delta_{n,-m}
$$

Theorem $1.1([1])$. Let $C_{\tau}$ be non-singular, then for any smooth tensor $f^{\lambda}(\sigma)$ of the weight $\lambda$ on $C_{\tau}$ the expansion (Fourier-type)

$$
f^{\lambda}(\sigma)=\sum_{n} f_{n}^{\lambda}(\sigma) \frac{1}{2 \pi i} \int_{C_{\tau}} f^{\lambda}\left(\sigma^{\prime}\right) f_{-n}^{1-\lambda}\left(\sigma^{\prime}\right)
$$

is valid. The same expansion is valid for the tensors $f^{\lambda}(z)$ which are holomorphic in the Riemann annulus $C_{\tau_{1} \tau_{2}}$ (Laurent-type expansion). (The theorem is valid for singular contours $C_{\tau}$ but smoothness conditions in this cases are slightly more rigourous. The theorem is valid independently of whether the $C_{\tau}$ contour is connected or not).

The proof of this theorem is based on the connection of the bases $f_{n}^{\lambda}$ with the "well-known discrete Baker-Akhiezer functions" in the soliton theory (about the details see [3] and [9]).

Remark. Recently the construction of the Fourier-Laurent-type bases for general multi-string diagram and the proof of theorem 1.1 for them was obtained by one of the authors with the help of the discrete Baker-Akhiezer vertor-functions. These functions appeared in the theory of the commutative difference operators with the matrix coefficients. ([10]). We shall give in the appendix the definition of the correspondence bases in the case $p_{i}= \pm 1, i=1, \ldots, 2 m$. 
The important properties of our bases (which immediately follow from the definition) are their almost-graded structure in respect to the multiplication

$$
f_{n}^{\lambda} f_{m}^{\mu}=\sum_{|k| \leqslant g / 2} Q_{n, m}^{\lambda \mu k} f_{n+m-k}^{\lambda+\mu}
$$

and in respect to the action of the vector-fields

$$
\left[e_{n}, f_{m}^{\lambda}\right]=\sum_{|k| \leqslant g_{0}} R_{n, m}^{\lambda k} f_{n+m-k}^{\lambda}, \quad g_{0}=\frac{3 g}{2} .
$$

(Here and below we shall use the special notations for bases for the $\lambda=-1,0$, $1 / 2,1,2$ :

$$
\left.e_{n}=f_{n}^{-1}, A_{n}=f_{n}^{0}, f_{n}^{1 / 2}=\varphi_{n}, f_{n}^{1}=d \omega_{-n}, f_{n}^{2}=d^{2} \Omega_{-n} .\right)
$$

For exceptional cases $\lambda, \mu,=0,1,|n|$ or $|m| \leqslant g / 2$ the sums in (1.6, 1.7) must include the additional terms. But in any case the sums include the terms only for $|k| \leqslant N$, where $N$ depends on $g, \lambda, \mu$, and doesn't depend on the $n, m$. This property leads us to the definition of the almost-graded algebras and modules.

Definition. The almost-graded ( $N$-graded) algebra $L$ (or module $M$ over $L$ ) is the algebra (or module) which can be expanded into the direct sum of the subspaces

$$
L=\sum_{i} L_{i}, \quad M=\sum_{i} M_{i}
$$

so that

$$
L_{i} L_{j} \subset \sum_{|k| \leqslant N} L_{i+j-k} ; \quad L_{i} M_{j} \subset \sum_{|k| \leqslant N_{1}} M_{i+j-k} .
$$

The basis $A_{n}=f_{n}^{0}$, as it follows from (1.6) with $\lambda=\mu=0$, defines the almostgraded structure in the commutative algebra $A^{\Gamma}$ of the meromorphy on $\Gamma$ functions which are holomorphic everywhere except the points $P_{ \pm}$.

The functions $A_{n}$ with $\pm n>g / 2$, as it follows from (1.3), are holomorphic in the neighbourhood of the points $P_{ \pm}$respectively and have at least the simple zero in these points. The choice of the base functions $A_{n}$ for $|n|<g / 2$ is not canonical. But in any case we can do it in such a way that

$$
A_{-g / 2}=1
$$

and the other functions $A_{n},-g / 2<n \leqslant g / 2$ have the poles in both points $P_{ \pm}$.

The analogue of the Heizenberg algebra is the algebra which is generated by the elements $\alpha_{n}, t$ with the following commutative relations

$$
\left[\alpha_{n}, \alpha_{m}\right]=\gamma_{n m} \cdot t, \quad \gamma_{n m}=\frac{1}{2 \pi i} \int_{C_{\tau}} A_{m} d A_{n}, \quad\left[\alpha_{n}, t\right]=0 .
$$

As it follows from (1.3), we have for $|n|>g / 2,|m|>g / 2$ that

$$
\gamma_{n m}=0, \quad|n+m|>g .
$$

For all values of $n, m$ the slightly weak condition is held: $\gamma_{n m}=0$ if $|n+m|>2 g$. In the particular case: $g=0, P_{+}=0, P_{0}=\infty$ the definition (1.12) leads to the 
ordinary Heizenberg algebra of the creation and annihilation operators of the free closed bosonic string.

In the case $\lambda=-1$ we have the almost-graded algebra $\mathcal{L}^{\Gamma},\left(e_{n}=f_{n}^{-1}\right)$

$$
\left[e_{n}, e_{m}\right]=\sum_{k=-g_{0}}^{g_{0}} C_{n m}^{k} e_{n+m-k}
$$

The analogue of the Virasoro-type algebra is the central extended algebra $\hat{\mathcal{L}}^{\Gamma}$ with the basis $e_{n} \cdot t$ with the commutative relations:

$$
\left[e_{n}, e_{m}\right]=\sum_{k=-g_{0}}^{g_{0}} c_{n m}^{k} e_{n+m-k}+\chi_{n m} \cdot t, \quad\left[e_{n}, t\right]=0
$$

where the cocycle $\chi_{n m}=\chi\left(e_{n}, e_{m}\right)$ is defined by the formula

$$
\left.\chi(f, g)=\frac{1}{48 \pi i} \int_{C_{\tau}}\left(f^{\prime \prime \prime} g-g^{\prime \prime \prime} f\right)-2\left(f^{\prime} g-g^{\prime} f\right) \times R\right) d z .
$$

Here $f=f(z) \partial / \partial z, g=g(z) \partial / \partial z$ vector-fields, $R(z)$-projective connection which is holomorphic on $\Gamma$ except the points $P_{ \pm}$. (The projective connection is the value $R(z)$ which is transformed by the following way

$$
R(w)=R(z)\left(w^{\prime}\right)^{2}+\left(\frac{w^{\prime \prime \prime}}{w^{\prime}}-\frac{3}{2}\left(\frac{w^{\prime \prime}}{w^{\prime}}\right)^{2}\right), \quad w^{\prime}=\frac{d w}{d z}
$$

in respect to the transformation of local system of the co-ordinate: $w=w(z)$. If the projective connection is holomorphic in the points $P_{ \pm}$also then

$$
\chi_{n m}=0, \quad \text { if }|n+m|>3 g .
$$

Remark. The central extensions of the algebras $A^{\Gamma}$ and $\mathcal{L}^{\Gamma}$ can be defined for any closed contour $\gamma$ on $\Gamma\left(P_{+} \cup P_{-}\right)$by the previous formula if one changes in them the contours $C_{\tau}$ for $\gamma$.

\section{Conjecture.}

$$
H^{2}\left(\mathcal{L}^{\Gamma}, R\right)=H_{1}\left(\Gamma \backslash\left(P_{+} \cup P_{-}\right), R\right) .
$$

Theorem $([1,2])$. The cohomology class of contours $\left[C_{\tau}\right]$ is one and only one cohomology class of contours $\gamma$ such that corresponding central extensions of algebras $A^{\Gamma}$ and $\mathcal{L}^{\Gamma}$ are also almost graduated.

\section{Riemann analogues of Heizenberg and Virasoro algebras IN STRING THEORY}

Let $X^{\mu}(Q)$ and $P^{\nu}(Q), Q \in \Gamma$, be the operator-valued scalar function and 1form in respect to the variable $Q$ on $\Gamma$. They commute with each other in different moments of time (i.e. $\tau(Q) \neq \tau\left(Q^{\prime}\right)$ ) and satisfy the commutative relations

$$
\left[X^{\mu}(Q), P^{\nu}(Q)\right]=-i \eta^{\mu \nu} \Delta_{\tau}\left(Q, Q^{\prime}\right), \quad \text { if } \tau(Q)=\tau\left(Q^{\prime}\right)=\tau .
$$


Here $\Delta_{\tau}\left(Q, Q^{\prime}\right)$ is the " $\delta$-function" on the contour $C_{\tau}$ (it is function in respect to $Q$ and 1-form in repect to $Q^{\prime}$; for any smooth function $f(Q)$ we have by definition

$$
\left.f(Q)=\frac{1}{2 \pi i} \int_{C_{\tau}} f\left(Q^{\prime}\right) \Delta_{\tau}\left(Q, Q^{\prime}\right)\right) .
$$

$\eta^{\mu \nu}=\eta^{\mu} \delta_{\mu}^{\nu}$ the metric of the physical space (Minkovskii or Euclidean), $\mu=$ $1, \ldots, \mathcal{D}$.

In the work [2] it was proved that the coefficients of the expansion

$$
\mathcal{I}^{\mu}(\sigma)=\partial_{\sigma} X^{\mu} d \sigma+\pi P^{\mu}(\sigma)=\sum \alpha_{n}^{\mu} d \omega_{n}(\sigma)
$$

$\left(d \omega_{n}=f_{-n}^{1}\right)$ satisfy the commutative relations of the Heizenberg-type algebra

$$
\left[\alpha_{n}^{\mu}, \alpha_{m}^{\nu}\right]=\eta^{\mu \nu} \gamma_{n m} .
$$

The coefficients $\bar{\alpha}_{n}^{\mu}$ of the expansion

$$
\overline{\mathcal{I}}^{\mu}(\sigma)=X_{\sigma}^{\mu} d \sigma-\pi P^{\mu}=\sum \bar{\alpha}_{n}^{\mu} \overline{d \omega_{n}}(\sigma)
$$

satisfy the commutative relations of the conjugate Heizenberg-type algebra

$$
\left[\bar{\alpha}_{n}^{\mu}, \bar{\alpha}_{m}^{\nu}\right]=\bar{\gamma}_{n m} \eta^{\mu \nu} .
$$

At the same time ([2])

$$
\left[\alpha_{n}^{\mu}, \bar{\alpha}_{m}^{\nu}\right]=0 .
$$

That's why we can restrict ourselves and can consider only the holomorphic part of the theory. The full Fock space would be as usual a tensor product of the holomorphic and antiholomorphic parts.

The holomorphic parts of the "vacuum-sectors" of in - and out-Fock spaces are defined as the spaces, which are generated by the left and right actions of the operators $\alpha_{n}^{\mu}$ from the vacuum-vectors $|0\rangle$ and $\langle 0|$

$$
\begin{gathered}
\alpha_{n}^{\mu}|0\rangle=0, \quad n>g / 2, n=-g / 2, \\
\langle 0| \alpha_{n}^{\mu}=0, \quad n \leqslant-g / 2 .
\end{gathered}
$$

According to the previous definitions this means that the vacuum-vectors are annihilated by the operators, corresponding to the basic functions $A_{n}$ which are holomorphic in the neighbourhoods of the points $P_{+}$(for $\left.|0\rangle\right)$ and $P_{-}$(for $\langle 0|$ ).

It can be shown that such defined spaces $H_{\Gamma}^{ \pm}$(which of course depend on $P_{ \pm}$ also) are isomorphic to the vacuum sectors of the ordinary Fock spaces for small circles around $P_{ \pm}$. More precisely, let $a_{N, t}^{\mu}, N \in Z$, be the operators with the ordinary commutative relations

$$
\left[a_{N, \pm}^{\mu}, a_{M, \pm}^{\nu}\right]=\eta^{\mu \nu} N \delta_{N,-M} .
$$

Then it can be easily checked that the operators $\alpha_{n}^{\mu}$ which are defined for $|n|>g / 2$ by both of the formulae (2.9) and in the slightly different form for $|n| \leqslant g / 2$ satisfy the commutator relations (2.5).

$$
\alpha_{n}^{\mu}=\sum_{s=0}^{\infty} \xi_{s}^{+}(n) a_{n+s-g / 2}^{\mu,+}=\sum_{s=0}^{\infty} \xi_{s}^{-}(n) a_{s-n-g / 2}^{\mu,-}
$$


where

$$
A_{n}=z_{ \pm}^{ \pm n-g / 2} \sum_{s=0}^{\infty} \xi_{s}^{ \pm}(n) z_{ \pm}^{s}
$$

The conditions (2.7) do coincide with the ordinary one

$$
a_{N,+}^{\mu}|0\rangle=0, \quad\langle 0| a_{N,-}^{\mu}, \quad N \geqslant 0
$$

which define the "local" Fock spaces.

Remark. As it was mentioned by A. Polyakov (private talk) the global definition of the basic function $A_{n}$ and the corresponding possibility to express them in terms of both local systems of co-ordinates $z_{ \pm}$near the points $P_{ \pm}$must contain in some sense the information about the scattering process of the string exitations.

Of course, one can define using (2.9) the formal Bogoluobov transformation

$$
a_{N,-}^{\mu}=\sum_{n}\left(U_{-}\right)_{N, n}^{-1}\left(U_{+}\right)_{n M} a_{M,+}^{\mu}
$$

where matrix elements $\left(U_{ \pm}\right)_{n M}$ are given by the right hand side of the equalities (2.9). But it looks as if even for each $N, M$ the corresponding series in r.h.s. of (2.11) diverge. Up to now the question of the regularization of (2.11) is not exactly clear.

In the classical case the densities of the Hamiltonian and momentum are equal to the sum and difference of the values

$$
T(z)=\frac{1}{2} \mathcal{I}^{2}(z), \quad \bar{T}(z)=\frac{1}{2} \overline{\mathcal{I}}^{2}(z) .
$$

The definitions of the corresponding quantum operators require, as usual, the definition of the "normal ordering", of the products $\alpha_{n}^{\mu} \alpha_{m}^{\nu}$. There exists the arbitrariness in this definition because operators $\alpha_{n}^{\mu}, \alpha_{m}^{\nu}$ don't commute for $|n|,|m| \leqslant g / 2$.

Let's dissect the integer (or half-integer) plane of pairs $(n, m)$ into two parts $\sum^{ \pm}$ such that $\sum^{+}$differs from tht integer half-plane $m \leqslant n$ only in the finite number of points. The definition of the normal ordering depends on the choice of $\sum^{ \pm}$

$$
: \alpha_{n} \alpha_{m}:=\alpha_{n} \alpha_{m}, \quad(n, m) \in \sum^{+} ; \quad: \alpha_{n} \alpha_{m}:=\alpha_{m} \alpha_{n}, \quad(n, m) \in \sum^{-} .
$$

As it follows from (2.7) the operator

$$
T(Q)=\frac{1}{2}: \mathcal{I}^{2}(Q):=\frac{1}{2} \sum_{n, m}: \alpha_{n} \alpha_{m}: d \omega_{n}(Q) d \omega_{m}(Q)
$$

is correctly defined. It is quadratic differential on $\Gamma$. That's why it can be expanded in the form

$$
\begin{gathered}
T(Q)=\sum_{k} L_{k} d^{2} \Omega_{k}(Q) . \quad d^{2} \Omega_{n}=f_{-n}^{2} \\
L_{k}=\frac{1}{2} \sum_{n, m} l_{n m}^{k}: \alpha_{n} \alpha_{m}:, \quad l_{n m}^{k}=\frac{1}{2 \pi i} \int_{C_{\tau}} e_{k} d \omega_{n} d \omega_{m} .
\end{gathered}
$$


If $|k|>g_{0}$

$$
l_{n m}^{k}=0, \quad|n+m-k|>g / 2 .
$$

For $|k| \leqslant g_{0}$ the width of the strip in the plane of the pairs $(n, m)$, such that $l_{n m}^{k}$ may differ from zero, is slightly larger. But in any case it remains finite.

Theorem 2.1 ([2]). The operators $e_{k}=-L_{k}$, where $L_{k}$ are given by the formula (3.13), (3.15), satisfy the commutator relations (1.15) of the Riemann analogues of the Virasoro algebra with the central charge $t=\mathcal{D}$. The cocycle $\chi_{n m}=\chi_{n m}^{\Sigma}$ depends on the choice of the normal ordering but his cohomology class does not depend on this choice.

We shall call the normal ordering admissible if it corresponds to the choice of $\sum^{ \pm}$ such that $\sum^{+}$differs from the half plane $n \leqslant m$ only in the strip $|n+m| \leqslant g-2$. The corresponding projective connection $R_{\Sigma}$ (which defines $\chi_{n m}^{\Sigma}$ ) is holomorphic on $\Gamma$ everywhere (including the points $P_{ \pm}$). For admissible normal ordering the following important conditions of the regularity of vacuum

$$
L_{k}|0\rangle=0, \quad k \geqslant g_{0}-1, \quad\langle 0| L_{k}=0, \quad k \leqslant-g_{0}+1
$$

are fulfilled (details see [3]).

Remark. The quadratic expressions of the form (2.16) are well-known in the case of genus $g=0$. They are the special case of the Sugawara-construction of the Virasoro generators through the generators of the Kac-Moody algebras. The Riemann analogues of the untwisted Kac-Moody algebras was proposed in [1]. The generalization of the Sugawara construction for the case of the Riemann surfaces of the genus $g>0$ was proposed in [12].

The projective connection $R_{\Sigma}$ corresponding to the co-cycle $\chi_{n m}^{\Sigma}$ depends, as well as the tensor $T(z)=T_{\Sigma}(z)$, on the choice of the normal ordering. But the operator-valued projective connection

$$
\tilde{T}(z)=T_{\Sigma}(z)+\frac{\mathcal{D}}{2} R_{\Sigma}
$$

doesn't depend on this choice and is a canonically defined pseudo-tensor of the energy-momentum. The last statement follows from the theorem.

Theorem $2.2([3])$. The chronological product $\mathcal{I}(z) \mathcal{I}(w)=\mathcal{I}^{\mu}(z) \eta^{\mu \nu} \mathcal{I}^{\nu}(w)$, where $\tau(z)>\tau(w)$ is correctly defined. For $z \rightarrow w$ the following expansion is valid

$$
\mathcal{I}(z) \mathcal{I}(w)=\mathcal{D} \frac{d z d w}{(z-w)^{2}}+2 \tilde{T}(z)+O(z-q) .
$$

For the pseudo-tensors of the energy-momentum on the arbitrary Riemann surfaces the ordinary operator expansions are fulfilled.

Theorem 2.3 ([3]). The chronological product $\tilde{T}(z) \tilde{T}(w), \tau(z)>\tau(w)$ is correctly defined. For $z \rightarrow w$ we have

$$
\tilde{T}(z) \tilde{T}(w)=\frac{\mathcal{D}}{2(z-w)^{4}}+\frac{2 \tilde{T}(z)}{(z-w)^{2}}+\frac{\tilde{T}_{z}(z)}{z-w}+O(1) .
$$


The definition of the in - and out-Fock spaces, the construction of the globally defined operator fields on $\Gamma, \mathcal{I}(z), \tilde{T}(z)$ is only the first part of our program. The following important step is the construction of the bilinear product (coupling) between the spaces $H_{\Gamma}^{ \pm}$. The conformal invariance requires that the operators $L_{k}$ must be formally self-adjoint in respect to this coupling, i.e.

$$
\left\langle\varphi \mid L_{k} \psi\right\rangle=\left\langle\varphi L_{k} \mid \psi\right\rangle=\left\langle\varphi\left|L_{k}\right| \psi\right\rangle, \quad \varphi \in H_{\Gamma}^{-}, \psi \in H_{\Gamma}^{+} .
$$

It is clear that these conditions would be fulfilled in the case when

$$
\left\langle\varphi \mid \alpha_{n}^{\mu} \psi\right\rangle=\left\langle\varphi \alpha_{n}^{\mu} \mid \psi\right\rangle=\left\langle\varphi\left|\alpha_{n}^{\mu}\right| \psi\right\rangle \text {. }
$$

In the case $g=0$ the conditions $(2.22)$ and the condition $\langle 0 \mid 0\rangle=1$ uniquely define the product $\langle\varphi \mid \psi\rangle$ of any elements of the vacuum sectors of the in - and out-Fock spaces. In the general case $g>0$, these conditions are insufficient because the operators $\alpha_{n}^{\mu}$ with $-g / 2<n \leqslant g / 2$ don't annihilate neither in-nor out-vacuum vectors.

\section{BLOCH'S HALF-DIFFERENTIALS AND "FERMIONIZATION FORMULAE"}

Let's fix the contour $\sigma$ on $\Gamma$, connecting the points $P_{ \pm}$, and the unitary representation of the fundamental group $\rho: \pi_{1}(\Gamma) \rightarrow S^{1}=\{z \in C,|z|=1\}$. If we fix on $\Gamma$ the canonical basis of cycles $a_{i}, b_{j}$ with the intersection matrix $a_{i} \circ a_{j}=b_{i} \circ b_{j}=0$, $a_{i} \circ b_{j}=\delta_{i j}$, then this representation can be defined by the real numbers $u_{j}$, $j=1, \ldots, g: \rho\left(a_{j}\right)=\exp \left(2 \pi i u_{j}\right), \rho\left(b_{j}\right)=\exp \left(2 \pi i u_{g+j}\right)$.

Lemma 1. For the representation $\rho$ in the general position and for the fixed number $p$ there exists the unique half-differential $\varphi_{\nu}(z, \rho), \nu-p \in Z+1 / 2$, such that it is holomorphic on $\Gamma$, cutting along the cycles $a_{i}, b_{j}$ and the contour $\sigma$, everywhere except the points $P_{ \pm}$. In the neighbourhoods of these points it has the form

$$
\left.\varphi_{\nu}(z, \rho)=\varphi_{\nu, 1 / 2}^{ \pm} z_{ \pm}^{ \pm \nu-1 / 2}\left(1+O\left(z_{ \pm}\right)\right)\left(d z_{ \pm}\right)\right)^{1 / 2},
$$

$\varphi_{\nu, 1 / 2}^{ \pm} \equiv 1$. It is multiplied by $\rho(\gamma)$ when one goes around the cycle $\gamma$. Its boundary values $\varphi_{\nu, \pm}$ on the contour $\sigma$ are connected by the relation

$$
\varphi_{\nu,+}(t, \rho)=\exp (2 \pi i p) \varphi_{\nu,-}(t, \rho), \quad t \in \sigma .
$$

It can be mentioned that the representations $\rho$ such that $\rho(\gamma)= \pm 1$ for any $\gamma \subset \Gamma$ correspond to the spinor structures on $\Gamma$. The spinor structures are in the general position (in the sense of the statement of lemma) only if it is an even spinor structure.

Let's consider the ordinary Dirac fermionic operators $\psi_{\nu}, \psi_{n}^{+}, \nu-1 / 2 \in Z$, with the anti-commutator relations

$$
\left[\psi_{\nu}, \psi_{\mu}\right]_{+}=\left[\psi_{\nu}^{+}, \psi_{\mu}^{+}\right]_{+}=0, \quad\left[\psi_{\nu}, \psi_{\mu}^{+}\right]_{+}=\delta_{\nu+\mu, 0}
$$

The Fock spaces $\mathcal{H}^{ \pm}$of the Dirac fermions are generated by the operators $\psi_{\nu}, \psi_{\mu}^{+}$ from the vacuum vectors $\left|0_{F}\right\rangle,\left\langle 0_{F}\right|$

$$
\begin{array}{ll}
\psi_{\nu}\left|0_{F}\right\rangle=\psi_{\nu}^{+}\left|0_{F}\right\rangle=0, & \nu>0, \\
\left\langle 0_{F}\right| \psi_{\nu}=\left\langle 0_{F}\right| \psi_{\nu}^{+}=0, & \nu<0 .
\end{array}
$$


Let's introduce the "fermionic" fields

$$
\begin{gathered}
\psi(z, \rho)=\sum_{\nu} \psi_{\nu} \varphi_{-\nu}(z, \rho), \quad \nu-1 / 2 \in Z . \\
\psi^{+}(z, \rho)=\sum_{\nu} \psi_{\nu}^{+} \varphi_{-\nu}^{+}(z, \rho), \quad \varphi_{\nu}^{+}(z, \rho)=\varphi_{\nu}\left(z, \rho^{-1}\right) .
\end{gathered}
$$

Theorem 3.1. The chronological product $\psi(z, \rho) \psi^{+}(w, \rho), \tau(z)>\tau(w)$ is correctly defined. For $z \rightarrow w$

$$
\psi(z, \rho) \psi^{+}(z, \rho)=\frac{\sqrt{d z d w}}{z-w}+\mathcal{I}(z, \rho)+O(z-w) .
$$

The coefficients of the expansion

$$
\mathcal{I}(z, \rho)=\sum \alpha_{n}(\rho) d w_{n}(z), \quad n-g / 2 \in Z
$$

satisfy the commutator relations (1.12) (with $t=1$ ), i.e.

$$
\left[\alpha_{n}(\rho), \alpha_{m}(\rho)\right]=\gamma_{n m}
$$

of the Riemann analogue of the Heizenberg algebra.

Below we shall define the set of "Szego"-type kernels $S_{p}(z, w, \rho)$ which are holomorphic $1 / 2$-differential in respect to the variables $z, w$ on $\Gamma$, cutting along the cycles $a_{i}, b_{j}$ and contour $\sigma$, everywhere except the points $P_{ \pm}$and the diagonal $z=w$. For the fixed $w$ (resp. $z$ ) it is multiplied by $\rho(\gamma)\left(\operatorname{resp} . \rho^{-1}(\gamma)\right)$ when one goes around the cycle $\gamma$. The boundary values $S_{p}$ on $\sigma$ satisfy the relation

$$
S_{p}^{+}(t, w, \rho)=e^{2 \pi i p} S^{-}(t, w, \rho), \quad S^{+}(z, t, \rho)=e^{-2 \pi i p}(z, t, \rho), \quad t \in \sigma .
$$

In the neighbourhoods of the points $P_{ \pm}$we have

$$
\begin{aligned}
& S_{p}\left(z_{ \pm}, w, \rho\right)=z_{ \pm}^{\mp p} O(1)\left(d z_{ \pm}\right)^{1 / 2} \\
& S_{p}\left(z, w_{ \pm}, \rho\right)=w_{ \pm}^{ \pm p} O(1)\left(d w_{ \pm}\right)^{1 / 2} .
\end{aligned}
$$

The last condition, which uniquely defines the kernel $S_{p}$, requires that near the diagonal it has the form

$$
S_{p}(z, w, \rho)=\frac{\sqrt{d z d w}}{z-w}+d s_{p}(z, \rho)+O(z-w) .
$$

It can be shown that $d s_{p}(z, \rho)$ is the single-valued holomorphic differential except the points $P_{ \pm}$where it has the simple poles with the residues $\pm p$.

Corollary. The following formula ("ferminization")

$$
\alpha_{n}(\rho)=\sum_{\nu, \mu} a_{\nu, \mu}^{n}: \psi_{\nu} \psi_{-\mu}^{+}:+a_{n}
$$

gives the representation of the Heizenberg-type algebra in the spaces $\mathcal{H}^{ \pm}$. Here the coefficients

$$
a_{\nu, \mu}^{n}=\frac{1}{2 \pi i} \int_{C_{\tau}} \varphi_{-\nu} \varphi_{\mu}^{+} A_{n}, \quad a_{n}=\frac{1}{2 \pi i} \int_{C_{\tau}} A_{n} d s_{0}
$$


depend on the representation $\rho$. From (1.3), (3.1), it follows that

$$
a_{\nu, \mu}^{n}=0, \quad|n|>g / 2, \quad|n-\nu-\mu|>g / 2 .
$$

In the case of $|n| \leqslant g / 2$ the "strip" in the $(\nu, \mu)$-plane, outside $a_{\nu, \mu}^{n}=0$, becomes slightly bigger, but it is less than $|n-\nu-\mu| \leqslant g$.

The differential $d s_{0}(z, \rho)$ is holomorphic. Hence

$$
a_{n}=a_{n}(\rho)=0, \quad|n|>g / 2, n=-g / 2 .
$$

The "ferminization formulae" give the possibility to introduce the "coupling" between $H_{\Gamma}^{ \pm}$using the natural coupling between the fermionic Fock spaces $\mathcal{H}^{ \pm}$. The latter is uniquely defined by the requirements

$$
\begin{gathered}
\langle\varphi \mid \psi \Psi\rangle=\langle\varphi \psi \mid \Psi\rangle=\langle\varphi|\psi| \Psi\rangle, \quad \varphi \in \mathcal{H}^{-}, \Psi \in \mathcal{H}^{+}, \\
\left\langle 0_{F} \mid 0_{F}\right\rangle=1 .
\end{gathered}
$$

Let the vectors $\left\langle 0_{F}|,| 0_{F}\right\rangle$ have the charge zero and the operator $\psi$ and $\psi^{+}$have the charge +1 and -1 respectively. Then

$$
\mathcal{H}^{ \pm}=\sum_{p} \mathcal{H}_{p}^{ \pm}, \quad p \in Z-\text { charge. }
$$

For any set of the representations $\rho_{\mu}, \mu=1, \ldots, \mathcal{D}$ in general positions, the correspondence

$$
\begin{gathered}
\alpha_{n}^{\mu} \rightarrow 1 \otimes \cdots \otimes \sqrt{\eta^{\mu}} \alpha_{n}\left(\rho_{\mu}\right) \otimes 1 \otimes \cdots \otimes 1 \\
|0\rangle=\bigotimes\left|0_{\rho_{\mu}}\right\rangle, \quad\langle 0|=\bigotimes\left\langle 0_{\rho_{\mu}}\right|
\end{gathered}
$$

where

$$
\left|0_{\rho}\right\rangle=Z_{+}(\rho)\left|0_{F}\right\rangle, \quad\left\langle 0_{\rho}\left|=Z_{-}(\rho)<0_{F}\right|\right.
$$

defines the isomorphism

$$
H_{\Gamma}^{ \pm} \cong\left(\mathcal{H}_{0}^{ \pm}\right)^{\otimes \mathcal{D}}
$$

The product of the constants $Z_{ \pm}(\rho)$ which is the norm of the vacuum vectors

$$
\left\langle 0_{\rho} \mid 0_{\rho}\right\rangle=Z_{+}(\rho) Z_{-}(\rho)\left\langle 0_{F} \mid 0_{F}\right\rangle=Z(\rho)
$$

can be naturally considered as the density of the partition function.

In the next paragraph we shall return to the problem of their definition. The normalized expectation values, i.e. the values of the form

$$
\langle H\rangle_{\rho}=\frac{\left\langle 0_{\rho}|H| 0_{\rho}\right\rangle}{\left\langle 0_{\rho} \mid 0_{\rho}\right\rangle}=\frac{\left\langle 0_{\rho}|H| 0_{\rho}\right\rangle}{Z(\rho)}
$$

don't depend on the constant $Z(\rho)$. Hence, we can consider them just now.

In the previous work $([3])$ it was proved that

$$
\left\langle\psi(z, \rho) \psi^{+}(w, \rho)\right\rangle_{\rho}=S_{0}(z, w, \rho) .
$$

In the modern physical literature the formula (3.23) is playing the role of the definition of the propagator of the free fermionic fields (in the case $\rho(\gamma)= \pm 1$ ) without any constructions of the proper fields. 
The calculations of the normalized expectation values of the products of the operators can be easily done using the standard Vick-formula, the correctness of which in our theory was proved in [3].

For example,

$$
\langle\mathcal{I}(z) \mathcal{I}(w)\rangle_{\rho}=-S_{0}(z, w, \rho) S_{0}(w, z, \rho) .
$$

When $z \rightarrow w$ the r.h.s. of this equality has the form

$$
-S_{0}(z, w, \rho) S_{0}(w, z, \rho)=\frac{d s d w}{(z-w)^{2}}+2 R_{\rho}(z)+O(z-w),
$$

where $R_{\rho}(z)$ is the Szego projective connection. The comparison of $(3.25,3.24)$ and (2.19) gives immediately

$$
\langle\tilde{T}(z)\rangle_{\vec{\rho}}=\sum_{\mu=1}^{\mathcal{D}} R_{\rho_{\mu}}(z), \quad \vec{\rho}=\left(\rho_{1}, \ldots, \rho_{\mathcal{D}}\right) .
$$

Remark. It must be specially mentioned that the projective connection $R_{\rho}(z)$ for any $g$ does not depend on the punctures $P_{ \pm}$.

Example. $g=1$. Consider the elliptic curve $\Gamma$ with the periods $2 \omega=1,2 \omega^{\prime}=\tau$. The Szego-kernel, corresponding to the representation $\rho(1)=\exp \left(2 \pi i u_{1}\right), \rho(\tau)=$ $\exp \left(2 \pi i u_{2}\right)$ has the form

$$
S_{0}(z, w, \rho)=\frac{\sigma(z-w+x)}{\sigma(z-w) \sigma(x)} e^{y(z-w)} \sqrt{d z d w}
$$

where

$$
\begin{gathered}
x=x_{1}+i x_{2}, \quad y=y_{1}+i y_{2}, \\
y_{1}=-2 \eta x_{1}, \quad y_{2}=-2 \frac{\operatorname{Im} \eta^{\prime}}{\operatorname{Im} \tau} x_{2}, \\
u_{1}=\frac{x_{2}}{\operatorname{Im} \tau}, \quad u_{2}=-x_{1}+\frac{x_{2}}{2 \pi}\left(\operatorname{Re} \eta^{\prime}-\operatorname{Re} \tau \cdot \frac{\operatorname{Im} \eta^{\prime}}{\operatorname{Im} \tau}\right) .
\end{gathered}
$$

Here and below $\sigma, \zeta, \wp$-Weierstrass elliptic functions $\eta=\zeta(1 / 2), \eta^{\prime}=\zeta(\tau / 2)$.

From the substitution of (3.27) into the (3.25) we obtain that in the global plane system of co-ordinate on $\Gamma$

$$
R_{\rho}(z)=\frac{1}{2} \wp(x)(d z)^{2}
$$

There are three even spinor structures on $\Gamma$. They correspond to the following values of $x=\omega_{\alpha},\left(\omega_{1}=1 / 2, \omega_{2}=\tau / 2, \omega_{3}=\frac{\tau+1}{2}\right)$. In these cases the formula (3.29) coincides with the results of [13], which were obtained with the help of the conformal Ward identities. This coincidence was briefly mentioned in [3]; it was not stressed sufficiently that (3.29) coincides with the mean value of energy-momentum tensor, which was obtained in [13] for the free fermionic field on $\Gamma$ but not for the scalar field.

It is well-known that in the $c=1$ case there are many different conformal field theories. The most important question: is there the possibility to introduce the 
coupling between $H_{\Gamma}^{ \pm}$which corresponds to the scalar part of the bosonic string theory?

The calculations which was made recently by one of the authors [10] makes very reliable the following conjecture.

Conjecture. The normalized vacuum expectation values $\langle H\rangle_{0}$ for the scalar field theory are equal to the averaging values $\langle H\rangle_{\rho}$ in respect to all representations $\rho: \pi_{1}(\Gamma) \rightarrow S^{1} \subset C^{2}$, i.e.

$$
\begin{gathered}
\langle H\rangle_{0}=\int_{0}^{1} \ldots \int_{0}^{1} \prod_{i=1}^{2 g} d u_{i}\langle H\rangle_{\rho}, \\
\rho\left(a_{j}\right)=\exp \left(2 \pi i u_{j}\right), \quad \rho\left(b_{j}\right)=\exp \left(2 \pi i u_{j+g}\right) .
\end{gathered}
$$

We cite here only one example: $H=\tilde{T}(z)$ and $g=1$. In that case

$$
\langle\tilde{T}(z)\rangle_{0}=\frac{\mathcal{D}}{2} \int_{0}^{1} \int_{0}^{1} d u_{1} d u_{2} \wp(x)=\frac{\mathcal{D}}{2}\left(4 \eta-\frac{2 \pi}{\operatorname{Im} \tau}\right) .
$$

where $x$ is given by the formula (3.28). The r.h.s. is exactly the same which was obtained from the Ward identities in [13] for the scalar fields.

\section{The SEMI-INFINITE FORMS AND "THE PRINCIPLE OF THE NORMALIZATION"}

The formulae $(2.15,3.11)$ define the structure of the Verma modules over the algebra $\hat{\mathcal{L}}^{\Gamma}$ in the spaces $\mathcal{H}_{p}^{ \pm}$. These modules are the particular cases of the general Verma modules which were introduced in $[1,2]$. The geometrical realization of these modules, which was proposed there, is the following (in the case $g=0$ it was done in the work [14]).

Let's fix the number $p$ and the contour $\sigma$, connecting the points $P_{ \pm}$. In the general position, for any $n, n-p-g / 2 \in Z$, there exists the unique up to the constant factor tensor $f_{n}^{\lambda}$ of the weight $\lambda \in Z$ (if $\lambda$ is half-integer then it is necessary to fix also the representation $\left.\rho: \pi_{1}(\Gamma) \rightarrow S^{1}\right)$ such that a) it is holomorphic on $\Gamma$ except the points $P_{ \pm}$and the contour $\left.\sigma ; \mathrm{b}\right)$ in the neighbourhoods of the points $P_{ \pm}$ it has the form $(1.3)$; c) its boundary values on a are connected by the following relation

$$
f_{n,+}^{\lambda}=e^{2 \pi i p} f_{n,-}^{\lambda} .
$$

Consider the right and left semi-infinite forms - exterior products of the form

$$
\begin{array}{ll}
f_{S+p+n_{0}}^{\lambda} \wedge f_{S+p+n_{1}}^{l} \wedge \cdots \wedge f_{S+p+n_{k}}^{\lambda} \wedge \ldots & \text { right-form } \\
\cdots \wedge f_{-S+p+m_{-k}}^{\lambda} \wedge f_{S+p+m_{-1}}^{\lambda} \wedge f_{-S+p+m_{0}} & \text { left-form. }
\end{array}
$$

$S=S(\lambda, g)=g / 2-\lambda(g-1)$, such that the sequences $n_{0}<n_{1}<\ldots$, become stable from number on. This means for some $k_{0}$ we have $n_{k}=k$ if $k>k_{0}$. The spaces of the finite linear combinations of the basic vectors of the form (4.2) would be denoted by $M_{p, \lambda}^{ \pm}=M_{p, \lambda, \sigma}^{ \pm}$(the sign + and - for right and left semi-infinite forms respectively). In [1,2] it was proved that the spaces $M_{p, \lambda}^{ \pm}$are the Verma-modules over $\hat{\mathcal{L}}^{\Gamma}$. The generating (singular) vectors $\left|\psi_{\lambda, p}^{+}\right\rangle$and $\left\langle\psi_{\lambda, p}^{-}\right|$have the form (4.2) 
where the corresponding sequences of indices are $n_{i}=i, i=0,1, \ldots$ They satisfy the relations

$$
\begin{gathered}
L_{n}\left|\psi_{\lambda, p}^{+}\right\rangle=0, \quad n>g_{0}=\frac{3 g}{2}, \quad L_{g_{0}}\left|\psi_{\lambda, p}^{+}\right\rangle=h_{\lambda, p}^{+}\left|\psi_{\lambda, p}^{+}\right\rangle, \\
\left\langle\psi_{\lambda, p}^{-}\right| L_{n}=0, \quad n<-g_{0}, \quad\left\langle\psi_{\lambda, p}^{-}\right| L_{-g_{0}}=h_{\lambda, p}^{-}\left\langle\psi_{\lambda, p}^{-}\right| .
\end{gathered}
$$

The central charge of the corresponding representation is given by the well-known formula

$$
t=-12 \lambda^{2}+12 \lambda-2
$$

The highest weights equal $h_{\lambda, p}^{ \pm}=\frac{1}{2} p(2 \lambda+p-1)$.

There exists the natural "coupling" between the spaces of all right and left semiinfinite form

$$
W_{\lambda}^{ \pm}=\bigoplus_{p} W_{\lambda, p}^{ \pm}
$$

(may-be it will be better to write the direct integral of these spaces). For the basic forms $f \in W_{\lambda}^{+}, g \in W_{\lambda}^{-}$let's consider the product $f \wedge g$. If this infinite (in both directions) form coincides after the permutation with the standard form (the exterior product of all basic tensors:

$$
\left.\bigwedge_{n} f_{n}^{\lambda}, \quad n-p-g / 2 \in Z\right)
$$

then we define

$$
\langle f \mid g\rangle=(-1)^{\varepsilon},
$$

where $\varepsilon$ is the sign of the corresponding permutation. In other cases the product $\langle f \mid g\rangle=0$ would be equal to zero by the definition. The scalar products between basic elements define the scalar products of any elements $f \in W_{\lambda}^{+}, g \in W_{\lambda}^{-}$by the linearity. It is non-trivial between subspaces $W_{\lambda, p_{ \pm}}^{ \pm}$when

$$
S+p_{+}+(S+p-)=1 .
$$

Lemma ([1]). The operators $L_{n}$ acting in the spaces of right and left semi-infinite forms are self-ajoint in the respect to the "coupling" which was defined above (i.e. the equalities (2.21) are fulfilled).

In the works $[3,9]$ we have discussed in details the "regularity conditions" for the vacuum vectors. They require that such vectors $\left|0_{\lambda}\right\rangle,\left\langle 0_{\lambda}\right|$ have satisfied the equalities

$$
L_{n}\left|0_{\lambda}\right\rangle=0, \quad n \geqslant g_{0}-1 ; \quad\left\langle 0_{\lambda}\right| L_{n}=0, \quad n \leqslant-g_{0}+1
$$

It was proved that they are fulfilled for the vectors $\left|\psi_{\lambda, 0}^{+}\right\rangle$and $\left\langle\psi_{\lambda, 0}^{-}\right|$. Hence these vectors are proportional to $\left|0_{\lambda}\right\rangle$ and $\left\langle 0_{\lambda}\right|$ :

$$
\left|\psi_{\lambda, 0}^{+}\right\rangle=\left(Z_{\lambda}^{+}\right)^{-1}\left|0_{\lambda}\right\rangle, \quad\left\langle\psi_{\lambda, 0}^{-}\right|=\left(Z_{\lambda}^{-}\right)^{-1}\left\langle 0_{\lambda}\right| .
$$

Again as in the previous paragraph it arises the problem of the definition of these normalizing constant. 
We assume that the vacuum vectors, as in the case $g=0$, are equal to the exterior product of all non-negative powers of the local parameters. Then

$$
\begin{aligned}
& \left|O_{\lambda}\right\rangle=1 \wedge z_{+} \wedge z_{+}^{2} \wedge \ldots \quad \text { "in-vacuum", } \\
& \left\langle O_{\lambda}\right|=\cdots \wedge z_{-}^{2} \wedge z_{-} \wedge 1 \quad \text { "out-vacuum". }
\end{aligned}
$$

The basic tensor-fields $f_{n}^{\lambda}$ are defined by the conditions (1.3) up to the factor. There are two different types of their normalization. In the case of in-normalization when we fix $\varphi_{n, \lambda}^{+} \equiv 1$

$$
Z_{\lambda}^{+}=1, \quad\left(Z_{\lambda}^{-}\right)^{-1}=\prod_{n \leqslant-S(\lambda)}\left(\varphi_{n, \lambda}^{-}\right)
$$

and

$$
Z_{\lambda}=\left\langle 0_{\lambda} \mid 0_{\lambda}\right\rangle=Z_{\lambda}^{+} Z_{\lambda}^{-}\left\langle\Psi_{\lambda, 0}^{-} \mid \Psi_{\lambda, 0}^{+}\right\rangle
$$

(In the case of out-normalization when $\varphi_{n, \lambda}^{-} \equiv 1$ we have $Z_{\lambda}^{-}=1$, but as it can be seen below the product $Z_{\lambda}^{+} Z_{\lambda}^{-}$does not depend on the choice of in - or outnormalization. Below we shall always fix the innormalization.)

From (4.7) it follows that

$$
\left\langle 0_{\lambda} \mid 0_{\lambda}\right\rangle=0, \quad \text { if } \lambda \neq \frac{1}{2} .
$$

In the case $\lambda=1 / 2$ (the only case when $2 S(\lambda, g)=1$ all the quantities depend on the representation. For the brevity we shall denote vectors $\left|0_{1 / 2, \rho}\right\rangle,\left\langle 0_{1 / 2, \rho}\right|$ by $\left|0_{\rho}\right\rangle$ and $\left\langle 0_{\rho}\right|$ respectively. From $(4.11,4.12)$ it follows that

$$
\left\langle 0_{\rho} \mid 0_{\rho}\right\rangle=Z(\rho)=\prod_{n \leqslant-1 / 2}\left(\varphi_{n, 1 / 2}^{-}(\rho)\right)^{-1} .
$$

Remark. Here and below (till the end of this paragraph) the infinite products of the form (4.11) are considered formally. Later we shall argue the problem of their regularization.

The operators $\psi_{\nu}, \psi_{\nu}^{+}, \nu-1 / 2 \in Z$ have the representation in the spaces $W_{k, 1 / 2}^{ \pm}$, $k \in Z$

$$
\psi_{\nu} \rightarrow \varphi_{\nu} \wedge
$$

(the exterior multiplication of the semi-infinite form by $\varphi_{\nu}$ )

$$
\psi_{\nu}^{+} \rightarrow \frac{\partial}{\partial \varphi_{-\nu}}
$$

(the differentiation of the semi-infinite form in respect to $\varphi_{-\nu}$ ). This correspondence gives the isomorphism of $W_{1 / 2}^{ \pm}$and $\mathcal{H}^{ \pm}$which is consistent with the coupling between the right and left spaces. 
A few remarks about the ghost sector. The Polyakov-Faddeev-Popov ghostfields in the string theory have the tensor weights -1 and 2 and are fermionic. We shall define them by

$$
b(z)=\sum b_{n} d^{2} \Omega_{n}, \quad c(z)=\sum c_{n} e_{n}(z) .
$$

The coefficients $b_{n}, c_{n}$ have the ordinary anti-commutators

$$
\left[b_{n}, b_{m}\right]_{+}=\left[c_{n}, c_{m}\right]_{+}=0, \quad\left[b_{n}, c_{m}\right]=\delta_{n, m} .
$$

As it was shown in [15], the definitions of the stress-energy operator of the ghostfields and the operator of the BRST-sharge can be easily generalized for the case of the Riemann surfaces of the genus $g>0$ with the help of the bases which were introduced in $\S 1$.

The full Fock space includes the tensor product of the "physical" and "ghost" sectors. In particular, the vacuum vector has to be the tensor product of the "physical" and "ghost" vacuum vectors. The regularity conditions of the ghost vacuum have the form (see $[3,9])$

$$
\begin{array}{ccl}
b_{n}\left|0_{g h}\right\rangle=0, \quad n \geqslant g_{0}-1, \quad c_{n}\left|0_{g h}\right\rangle=0, & n<g_{0}-1, \\
\left\langle 0_{g h}\right| b_{n}=0, \quad n \leqslant-g_{0}+1, \quad\left\langle 0_{g h}\right| c_{n}=0, & n>-g_{0}+1 .
\end{array}
$$

Let's define the action of the operators $b_{n}, c_{n}$ in the spaces $W_{\lambda=2}^{ \pm}$with the help of multiplication and differentiation of the semi-infinite forms

$$
b_{n} \rightarrow f_{n}^{\lambda=2} \wedge \ldots, \quad c_{n} \rightarrow \frac{\partial}{\partial f_{n}} \lambda=2 .
$$

This representation with the correspondence $\left|0_{g h}\right\rangle=\left|0_{2}\right\rangle,\left\langle 0_{2}\right|=\left\langle 0_{g h}\right|$ defines the isomorphisms between $W_{2}^{ \pm}$and $H_{\Gamma, g h}^{ \pm}$. Hence the "coupling" between $W_{2}^{ \pm}$ defines the "coupling" between the in - and out - ghost's Fock spaces. From (4.13) it follows that $\left\langle 0_{g h} \mid 0_{g h}\right\rangle=\left\langle 0_{2} \mid 0_{2}\right\rangle=0$. The most simple non-zero expressions can be obtained only in the presence of the insertions. For example

$$
\begin{array}{ll}
g=0, & \left\langle 0_{2}\left|c_{-1} c_{0} c_{1}\right| 0_{2}\right\rangle=1 \\
g=1, & \left\langle 0_{2}\left|b_{1 / 2} c_{-4 / 2}\right| 0_{2}\right\rangle \\
g>1, & \left\langle 0_{2}\left|b_{-g_{0}+2} \ldots b_{-g_{0}-2}\right| 0_{2}\right\rangle
\end{array} \quad=\prod_{n=-\infty}^{-g_{0}-1}\left(\varphi_{n, 2}^{-}\right)^{-1} \neq 0
$$

The operators $b_{n}$ for $|n| \leqslant g_{0}-2, g>1$ correspond to the holomorphic quadratic differentials which are the basis of the co-tangent bundle over the modular space of the genus $g$ surfaces. That's why the square of the modulus of the value (4.21) defines the measure on the modular space. The connection of this measure with Polyakove-Belavin-Knizhnik measure is under consideration now.

Remarks about the states with non-zero momentum. The ground states with the momentums $p_{ \pm}=\left(p_{ \pm}^{\mu}\right)$ at the points $P_{ \pm}$are defined by the following conditions

$$
\begin{gathered}
\alpha_{n}^{\mu}\left|\vec{p}_{n}^{\mu}\right\rangle=0, \quad n>g / 2, \quad \alpha_{-g / 2}^{\mu}\left|\vec{p}_{+}\right\rangle=p_{+}^{\mu}\left|\vec{p}_{+}\right\rangle, \\
\left\langle\vec{p}_{-}\right| \alpha_{n}^{\mu}=0, \quad n<-g / 2, \quad\left\langle\vec{p}_{-}\right| \alpha_{-g / 2}^{\mu}=p_{-}^{\mu}\left\langle\vec{p}_{-}\right| .
\end{gathered}
$$


The corresponding spaces would be denoted by $H_{\Gamma}^{ \pm}$. Let's introduce for the fixed real number $p$ the spaces $\mathcal{H}^{ \pm}(p)$ which are generated by the operators $\psi_{\nu}, \psi_{-\mu}^{+}$, $\nu, \mu \in Z_{ \pm p+1 / 2}$. The generating vectors are defined by the conditions

$$
\begin{gathered}
\psi_{\nu}\left|p_{F}\right\rangle=0, \quad \nu>p, \quad \psi_{-\mu}^{+}\left|p_{\Gamma}\right\rangle=0, \quad \mu<p, \\
\left\langle p_{F}\right| \psi_{\nu}=0, \quad \nu<-p, \quad\left\langle p_{F}\right| \psi_{-\mu}^{+}=0, \quad-m>-p .
\end{gathered}
$$

If the vectors $\left|p_{F}\right\rangle$ and $\left\langle p_{F}\right|$ have the charges $p$ and the operators $\psi_{\nu}, \psi_{\nu}^{+}$, as before, have charges \pm 1 respectively then

$$
\mathcal{H}^{ \pm}(p)=\sum_{\kappa} \mathcal{H}_{\kappa}^{ \pm}(p), \quad \kappa-p \in Z
$$

The spaces $\mathcal{H}_{\kappa}^{ \pm}(p)$ and $\mathcal{H}_{\kappa}^{ \pm}\left(p^{\prime}\right)$ are naturally isomorphic to each other if $p-p^{\prime} \in Z$. Hence, we can use the notation $\mathcal{H}_{k}^{ \pm}$.

Let's define the operators $\alpha_{n}(p)$ in the spaces $\mathcal{H}_{p}^{ \pm}$

$$
\alpha_{n, p}(\rho)=\sum a_{\nu, \mu}^{n}: \psi_{\nu} \psi_{-\mu}^{+}: p+\alpha_{n, p}, \quad \nu, \mu \in Z-p-1 / 2
$$

where the coefficients $a_{\nu, \mu}^{n}$ again are given by the first of the formulae (3.12), and

$$
\alpha_{n, p}=\frac{1}{2 \pi i} \int_{C_{\tau}} A_{n} d s_{p}
$$

The symbol : : $p$ means the normal ordering in respect to the vectors $|p\rangle,\langle-p|$.

Theorem 4.1 ([3]). The operators $\alpha_{n, p}(\rho)$ satisfy the commutator relations $(3.7)$. Their actions on $\mathcal{H}_{\kappa}^{ \pm}(\rho), \mathcal{H}_{\kappa}^{ \pm}\left(p^{\prime}\right), p-p^{\prime} \in Z$ are the same as

$$
\alpha_{n, p}(\rho)=\alpha_{n, p^{\prime}}(\rho) \text {. }
$$

Their actions on the vectors $\left|p_{F}\right\rangle,\left\langle p_{F}\right|$ satisfy the conditions (4.22).

The vectors $\left|p_{F}\right\rangle$ and $\left\langle p_{F}\right|$ are annihilated by the operators $L_{n}$ for $n>g_{0}$ and $n<-g_{0}$, respectively and are the eigenvectors for $L_{g_{0}}$ and $L_{-g_{0}}$ with the eigenvalues corresponding to the conformal weights $p^{2} / 2$.

Corollary. The spaces $\left(\mathcal{H}_{\rho^{\mu}}^{ \pm}\right)^{\otimes \mathcal{D}}$ and $H_{\Gamma}^{ \pm}(\vec{p})$ are isomorphic. This isomorphism can be defined using the representations (4.25) for the set of characters $\rho_{1}, \ldots, \rho_{\mathcal{D}}$ and the correspondence

$$
\begin{array}{cc}
|\vec{p}\rangle \rightarrow \bigotimes\left|p_{\rho}^{\mu}\right\rangle, & \langle\vec{p}|=\bigotimes\left\langle p_{\rho}^{\mu}\right|, \\
\left|p_{\rho}\right\rangle=Z_{p}^{+}(\rho)\left|p_{F}\right\rangle, & \left\langle p_{\rho}\right|=Z_{p}^{-}(\rho)\left\langle p_{F}\right| .
\end{array}
$$

The constants $Z_{p}^{ \pm}(\rho)$ can be defined from the normalization principle. We obtain (for in-normalization) that $Z^{+} \equiv 1$ and

$$
Z_{p}(\rho)=Z_{p}^{-}(\rho)=\prod_{n \leqslant S+p}\left(\varphi_{n, 1 / 2}^{-}(\rho)\right)^{-1} .
$$

Hence, we obtain the following formula

$$
A\left(p, \Gamma, P_{ \pm}\right)=\frac{\left\langle-p_{\rho} \mid p_{\rho}\right\rangle}{\left\langle 0_{\rho} \mid 0_{\rho}\right\rangle}=\frac{1}{Z(\rho)} \prod_{n \leqslant-S+p}\left(\varphi_{n, 1 / 2}^{-}(\rho)\right)^{-1}
$$


For general $p$ the quantity (4.29) must be regularized, as well as (4.11). For the integer $p$ all factors in the ratio (4.30) except the finite number are cancelled. Hence, for $p>0$-integer

$$
A_{\rho}\left( \pm p, \Gamma, P_{ \pm}\right)=\prod_{\nu= \pm 1 / 2}^{ \pm p \pm 1 / 2}\left(\varphi_{\nu, 1 / 2}^{-}(\rho)\right)^{ \pm 1}, \quad p>0 .
$$

From the definition of $\varphi_{\nu, 1 / 2}^{-}$it follows that the quantity (4.31) depends on $P_{ \pm}$ as the tensor of the weight $p^{2} / 2$.

From the results of the soliton theory the formula

$$
\varphi_{\nu, 1 / 2}^{-}=\frac{\theta[\rho]\left((\nu-1 / 2)\left(A\left(P_{+}\right)-A\left(P_{-}\right)\right)\right)}{\theta[\rho]\left((\nu+1 / 2)\left(A\left(P_{+}\right)-A\left(P_{-}\right)\right)\right)} E^{-2 \nu}\left(P_{+}, P_{-}\right)
$$

follows, where $\theta[\rho]$-theta-function with characteristic corresponding to the representation $\rho ; E\left(P_{+}, P_{-}\right)$- Prym-form

$$
E^{-2}\left(P_{+}, P_{-}\right)=\frac{\theta^{2}[m]\left(A\left(P_{+}\right)-A\left(P_{-}\right)\right)}{\left(\sum_{i} \omega_{i}\left(P_{+}\right) \theta_{i}[m]\right)\left(\sum_{i} \omega_{i}\left(P_{-}\right) \theta_{i}[m]\right)} .
$$

Let's consider now the infinite product (4.14). Using the $\zeta$-funcitonal regularization for the product of the factors $E^{-2 \nu}\left(P_{+}, P_{-}\right)$we obtain the ordinary conformal anomaly which cancels the corresponding conformal anomaly for the ghost sector (it appears after the regularization of the (4.21)). Hence, we can introduce (without loss of generality) the local co-ordinates $z_{ \pm}$near $P_{ \pm}$such that $E^{2}\left(P_{+}, P_{-}\right)=1$. In the product (4.14) the numerator of each factor cancels the denominator of the next factor. That's why it naturally regularizes the infinite product (4.14) so that

$$
\left\langle 0_{\rho} \mid 0_{\rho}\right\rangle=\prod_{\nu \leqslant-1 / 2}\left(\varphi_{\nu, 1 / 2}^{-}\right)^{-1}=\theta[\rho](0) .
$$

The same regularization for the quantity (4.29) gives

$$
\left\langle-p_{\rho} \mid p_{\rho}\right\rangle=\theta[\rho]\left(p\left(A\left(P_{+}\right)-A\left(P_{-}\right)\right)\right) .
$$

For integer $p$ the ratio of (4.34) and 4.33) coincides with (4.31).

The regularization, which was proposed above, using the exact theta-functional formulae looks naturally but not the usual one. Their exist two ways for obtaining this regularization without using the exact formulae. First of all one can compute the logarithmic derivative in respect to the deformation along the modular space. In [10] these computations were done and it was proved that after the usual regularization the logarithmic derivatives of (4.14) satisfy the Ward identities.

The second way of the regularization, which is also discussed in [10], is based on the computation of the logarithmic derivatives in respect to the changing of the representation $\rho$. It must be specially emphasized that according to the variables $u_{i}$ (which define $\rho$ ) the values $\varphi_{n, 1 / 2}^{-}$satisfy the equations of the hierarchy of the twodimensional Toda lattice. It gives us the hope that the quantity of the form (4.14) can be expressed in terms of the spectral theory of the two-dimensional Schrödinger operators [6]. This possibility is under considerations now. 


\section{REFERENCES}

[1] No source?

[2] No source?

[3] No source?

Institut des Hautes Etudes Scientifiques, 35, route de Chartres, 91440 Bures-surYVETTE (France) 